\title{
How to measure diagnosis-associated information in virtual slides
}

\author{
Klaus Kayser ${ }^{1 *}$, Jürgen Görtler ${ }^{2}$, Stephan Borkenfeld ${ }^{3}$, Gian Kayser ${ }^{4}$ \\ From The 10th European Congress on Telepathology and 4th International Congress on Virtual Microscopy \\ Vilnius, Lithuania. 1-3 July 2010
}

\begin{abstract}
The distribution of diagnosis-associated information in histological slides is often spatial dependent. A reliable selection of the slide areas containing the most significant information to deriving the associated diagnosis is a major task in virtual microscopy. Three different algorithms can be used to select the appropriate fields of view: 1) Object dependent segmentation combined with graph theory; 2) time series associated texture analysis; and 3) geometrical statistics based upon geometrical primitives. These methods can be applied by sliding technique (i.e., field of view selection with fixed frames), and by cluster analysis. The implementation of these methods requires a standardization of images in terms of vignette correction and gray value distribution as well as determination of appropriate magnification (method 1 only). A principle component analysis of the color space can significantly reduce the necessary computation time. Method 3 is based upon gray value dependent segmentation followed by graph theory application using the construction of (associated) minimum spanning tree and Voronoi's neighbourhood condition. The three methods have been applied on large sets of histological images comprising different organs (colon, lung, pleura, stomach, thyroid) and different magnifications, The trials resulted in a reproducible and correct selection of fields of view in all three methods. The different algorithms can be combined to a basic technique of field of view selection, and a general theory of "image information" can be derived. The advantages and constraints of the applied methods will be discussed.
\end{abstract}

\section{Introduction}

Virtual microscopy which is the work with virtual slides can be performed in two different manners: 1 ) interactive virtual microscopy and 2) automated virtual microscopy $[1,2]$. Interactive virtual microscopy translates the pathologist's work with conventional glass slides into the digital world, and leaves all work on the microscope to the pathologist. It includes slide navigation, magnification, illumination, focus, etc. Some digital features might be added, especially the contemporary view of different slides, automated storage of areas of interest (with inbuilt expert consultation), or creation of labels. Automated virtual microscopy tries to transfer as many functions as possible to the computer with the final aim, that the system evaluates and proposes the most likely diagnoses [3-5]. Such a system must translate all items

\footnotetext{
* Correspondence: klaus.kayser@charite.de

${ }^{1}$ UICC-TPCC, Charite, Berlin, Germany

Full list of author information is available at the end of the article
}

of the pathologist's work into computerized algorithms. These have not necessarily to work in a fully compatible manner; however, they must contain modules that reflect to the corresponding pathologist's work [6,7]. These modules will probably work in a "time sequence order", and include in addition to statistical procedures and classifiers tools that provide a reproducible and constant image quality, object, structure, and texture related magnifications, image analysis procedures, and field of interest recognition programs.

We want to describe some basic ideas and information recognition algorithms in image analysis that can be used for field of view detection in virtual slides which is the position and size of image compartments that posses the strongest association with the underlying disease.

\section{Basic assumptions}

The pathologist's work is the evaluation of a diagnosis from a microscopic image, which is an image analysis
C Biomed Central

C 2011 Kayser et al; licensee BioMed Central Ltd. This is an open access article distributed under the terms of the Creative Commons Attribution License (http://creativecommons.org/licenses/by/2.0), which permits unrestricted use, distribution, and reproduction in any medium, provided the original work is properly cited. 
algorithm in combination with external (clinical) data $[1,8,9]$. The pathologist's view focuses on specific biological meaningful objects and their spatial arrangement (structure) which include a) normal objects (cells, nuclei, etc.), b) abnormal objects (cancer cells, etc.), c) external objects (bacteria, parasites, silica, etc.), d) preserved structure with abnormal cellular societies (inflammatory infiltrates, fibrosis, etc.), e) destroyed structure (granuloma, necrosis, etc.), and f) abnormal structures (adenocarcinoma, sarcomatous growth pattern) [10-13]. A diagnosis from a histological image can be evaluated by recognition and classification of the objects, the formed structures, and their spatial arrangement. It is useful to introduce different levels of structures in order to describe for example the infiltration of lymphocytes into a vascular wall (a vessel would be of higher order compared to a lymphocyte because a vessel is built by a cellular sociology including endothelial cells, smooth muscle cells, a basal membrane, etc.). The details of this concept have been described in Kayser et al. [10,14-16].

The term information is derived from the latin word informare which means "create by teaching", in other words a communication procedure between a source (image) and the (understanding) receiver (pathologist). Shannon has analyzed the specific conditions of information transfer and content $[17,18]$. According to his theory information limits the broad variety of reactions of an (understanding) receiver to only one or a few appropriate ones. In other words, information is a statistical property and can be analyzed by statistical methods. Shannon introduced the term entropy as principle measure of information, which is derived from classic thermodynamics $[17,18]$. Entropy is a measure of the distance of a statistical population from its end stage using Kolmogorov's axiomatic approach of non-overlapping elementary events that are characterized by a probability $0 \leq \mathrm{p} \leq 1$.

Entropies $(\mathrm{E}=\Sigma\{\mathrm{pi} * \ln (\mathrm{pi})\})$ of different systems can be simply added (Es $=\Sigma(\mathrm{Ei})$, if there exists no correlation between the elements of the different systems (so called strong chaos), otherwise the more general term of Tsallis entropy has to be used $(\mathrm{Es}=\Sigma(\mathrm{Eq} 1+\mathrm{Eq} 2)+(1-\mathrm{q})$ $\left.{ }^{*} \mathrm{Eq}(1)^{*} \mathrm{Eq}(2)\right)$ [19-21].

\section{Macro- and microstages}

The basic elements of a system characterized by pi might be equally distributed in the system's space, or agglutinate to certain formations which can be considered as a "subspace". They are called macrostages. One can define the macrostages as new (higher order) events, and calculate the entropy of the original system based upon the macrostages and their internal entropy [22,23]. The number of microstages gives the maximum number of potential macrostages. An illustrating example is shown in figure 1 which is described in detail in [22]. The letter $\{\mathrm{T}, \mathrm{H}, \mathrm{I}, \mathrm{S}\}$ are the microstages, and the words \{THIS, IS, ISIS\} form the macrostages. The sequence of the macrostages form the structure.

The calculated Shannon entropies of the macrostages within the system (This is Isis) result in:

This : [-0.92]; is: [-0.46]; Isis: [- 0.64]; $\Sigma=-2.02$

that of the total system without macrostages this is Isis $\}=-1.58$,

and based upon the marcostages alone $\{$ [this] [is] $[$ Isis $]\}=-1.08$

The calculated probability of the macrostages based upon their internal entropies results in:

$\mathrm{P}($ this $)=(1.92) / 5.02=0.38$

$\mathrm{P}(\mathrm{is})=(1.46) / 5.02=0.29$

$\mathrm{P}($ isis $)=(1.64) / 5.02=0.33$

This is Isis: $\mathrm{E}=\{0.38 * \ln 0.38+0.29 * \ln 0.29+0.33 * \ln$ $0.33=-1.09$

The differences between the macrostages are: $[-0.46]+$ $[+0.22]=-0.18$.

If we transform the sequence into the question:

Is this Isis? we will get: $[+0.46]+[-0.28]=+0.18$.

The calculation of the total entropy of the (macrostage) system depends upon its structure, or, in other words, the calculation of macrostage entropies can be applied in relation to internal structures, such as sequential arrangement or spatial relationships $[16,22,23]$.

\section{Entropy calculation in relation to histological images (virtual slides)}

The information of a histological image which a pathologist can derive depends on the presence and spatial arrangement of cells or nuclei respectively. The different cell types that are present in such a slide can be addressed to microstages, and the corresponding disease to macrostages respectively. The microscopic images, the associated diagnoses, and the analyzed microstages are shown in the figure 2 - figures 4 . All in all 15 different cell types, and 8 different diseases are taken into account (figure 5). The assumed cellular distributions are given in figure 6 , and the computed entropies are shown in figure 7. As expected, notable differences exist between the different images (diseases). They are, however, not striking between quite different diseases, for example between a small cell lung cancer and normal lung parenchyma. The computed entropies can obviously not directly be translated to the biological significance of the corresponding disease.

\section{How to refine the entropy approach?}

Definition of image associated macro- and microstages

All (interactive) diagnostic information of a digitized image is derived from biological meaningful objects 


\section{Example of entropy, microstages, macrostages and order}

- Consider the sentence: This is Isis.

- Number of microstages: 4 (different letters)

- Number of macrostages:3 (different words)

- Expression of macrostages $\{\mathbf{T}, \mathrm{H}, \mathrm{I}, \mathrm{S}\}$ :

$-\{1,1,1,1\} \rightarrow$ This

$-\{0,0,1,1\} \rightarrow$ is

$-\{0,0,2,2\} \rightarrow$ Isis.

-Shannon's entropy:

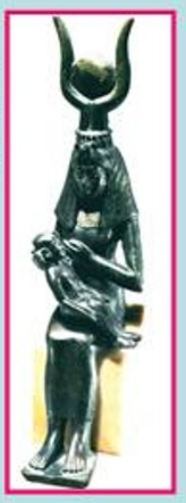

$$
S=-\left\{0.1^{\star} \ln (0.1)+0.4^{\star} \ln (0.4)\right\}^{*} 2=1.58
$$

- Total number of possible microstages: $4^{4}=256$

- Total number of possible macrostages:

\{(4+4-1)\}

$\{(4-1)\}=52$

Figure 1 Calculation of entropy, micro and macrostages of the sentence /This is Isis/.

Measure: Entropy $E=\boldsymbol{K}^{\star} \boldsymbol{Z}\left(\boldsymbol{p} \boldsymbol{i}^{*} \boldsymbol{I n}\right.$ pi) with $[y(p i)=1]$

$P i$ is the probability of a (micro) event to be seen in the stage $i$ (for example the number $<3>$ in a dye $(\mathrm{N}=6)$, or cell types in histological images.

Macrostages: set of images (normal, adenoma, carcinoma).
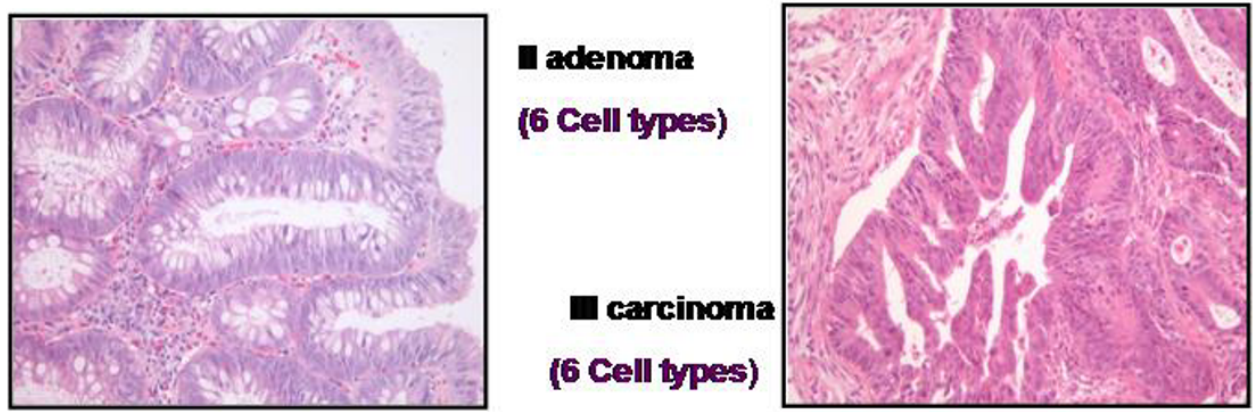

Figure 2 Example of cell types in histological images of colon diseases (normal, adenoma, and carcinoma) used for entropy calculation (Shannon and Tsallis). 


\section{Classification of images (macrostages)}

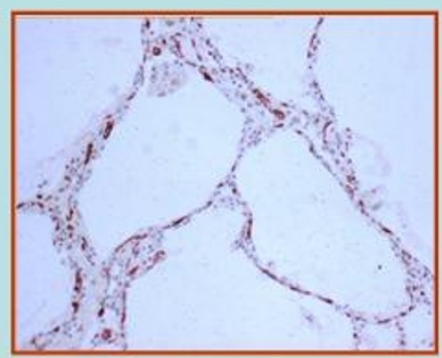

Normal

(4 Cell types)

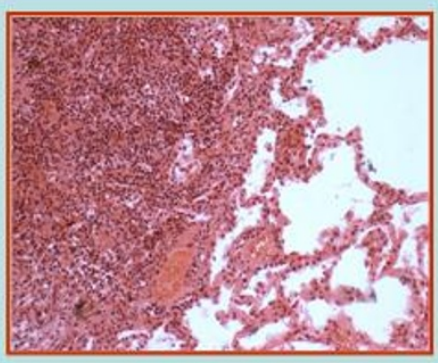

Bronchopneumonia

(8 Cell types)

Bronchitis

(6 Cell types)
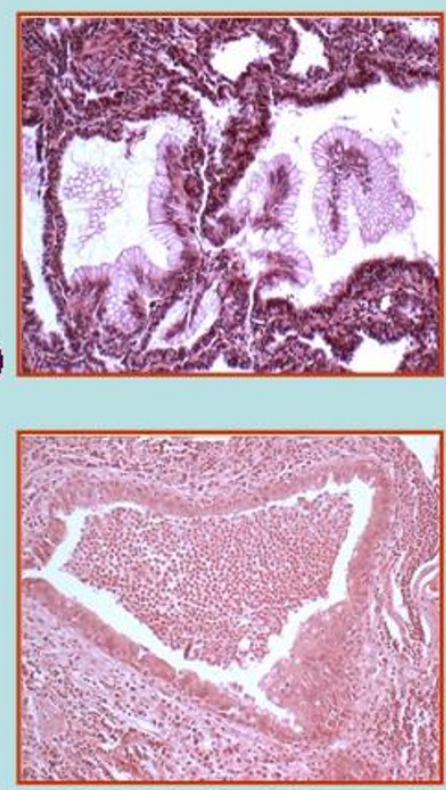

Figure 3 Example of cell types in histological images of lung diseases (normal, congenital adenomatoid malformation (CAM), bronchopneumonia, and bronchitis) used for entropy calculation (Shannon and Tsallis).

\section{Classification of images (macrostages)}

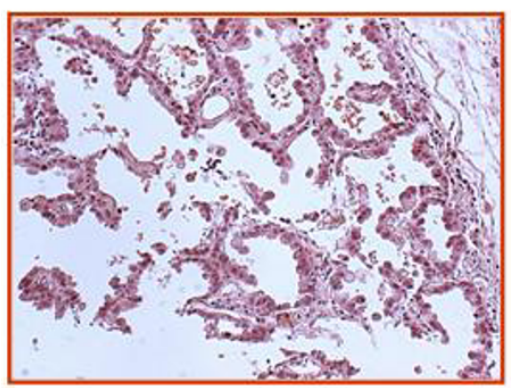

AАH

(6 Cell types)

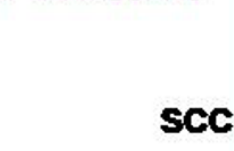

(5 Cell types)
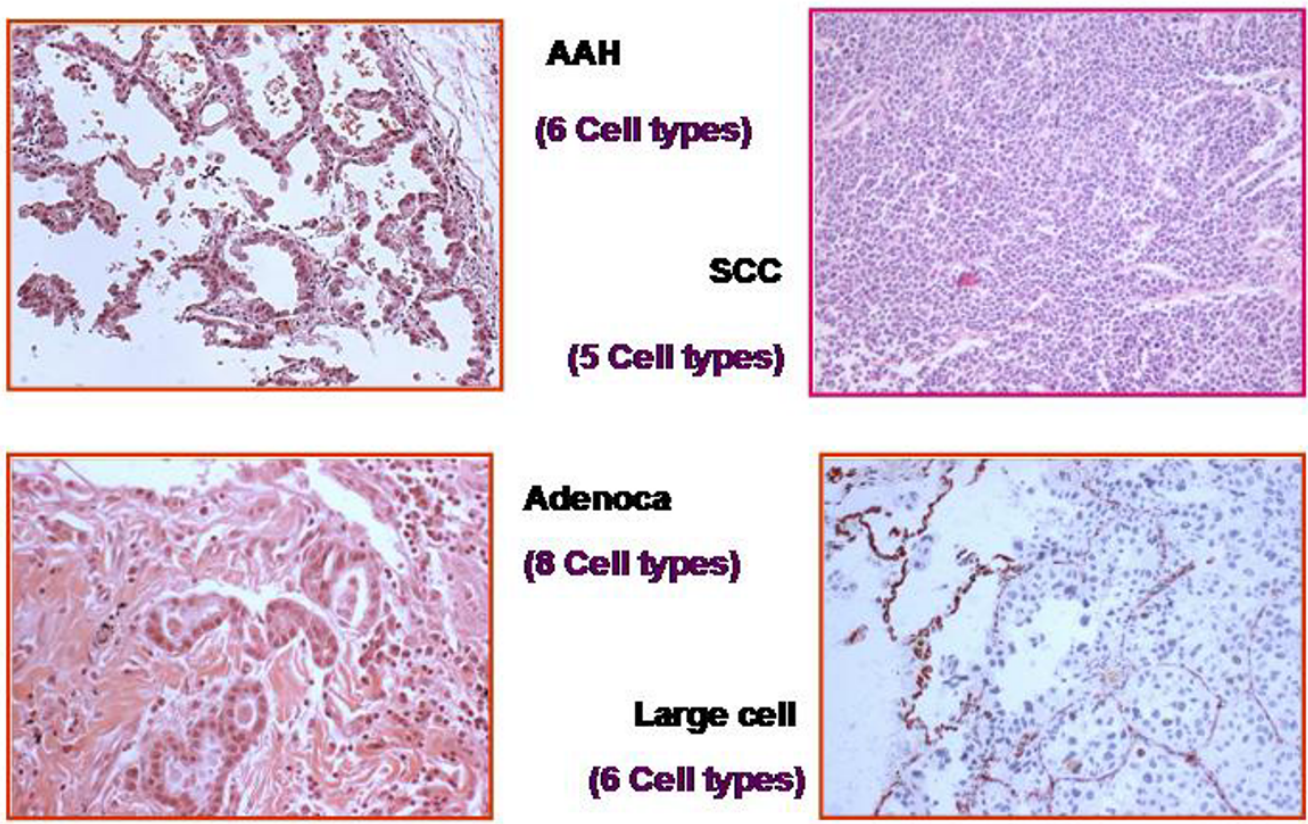

Adenoca

(8 Cell types)

Large cell

(6 Cell types)

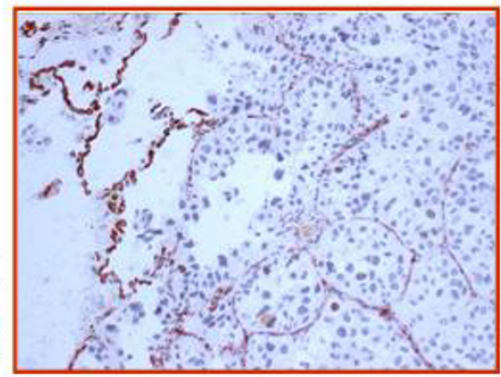

Figure 4 Example of cell types in histological images of lung diseases (atypical adenomatoid hyperplasia (AAH), small cell anaplystic carcinoma (SCC), Adenocarcinoma (Adenoca), and large cell anaplastic carcinoma (Large cell) used for entropy calculation (Shannon and Tsallis). 


\section{Diagnosis macrostages}

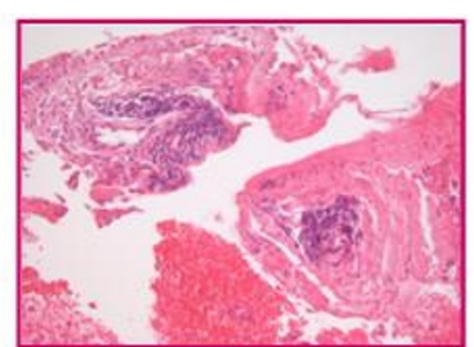

Diagnosis

Normal Lung

Congenital adenomatoid malformation

Bronchopneumonia

Bronchitis

Adenomatoid atypical hyperplasia

Adeno carcinoma

Large cell carcinoma

Small cell carcinoma
Analyzed cell types

$\{A 1, A 2, E, H\}$

\{C1, C2, E, Hi, Ly, PI, Eo\}

\{A1, A2, E, Hi, Ly, PI, Gr, Eo\}

\{Hi, Ly, PI, Gr, Eo, SQ\}

$\{E, H i$, Ly, PI, Eo, AH\}

\{A1, A2, E, Hi, Ly, PI, Eo, AC\}

\{A1, A2, E, Hi, Ly, LC\}

$\{E$, Ly, PI, Gr, SC\}

Figure $\mathbf{5}$ Used cell types for entropy calculation in relationship to the diagnosis (macrostage).

\section{Example of entropy, microstages,}

\section{macrostages, diagnosis}

Microstages (15): Pneumocytes (A1, A2), endothelliall cellls (E), histiocytes (Hi), lymphocytes (Ly), pllasma cells (PI), granulocybes (Gi), eosinophils (Eo), CAM1 cells (C1, C2), squamous metapllasila (SO), atypicall pneumocytes (AH), adeno carcinoma (AC), large celll carcinoma (LC), smalli celll carcinoma cells (SC):

[A1, A2, E, Hi, Ly, PI, Gr, Eo, C1,C2, SQ,AH,AC,LC, SC\}

Normal $\{50,05,40,05,00,00,00,00,00,00,00,00,00,00,00\}$

CAM $\{00,00,20,05,05,05,00,05,40,20,00,00,00,00,00\}$

Pneumonia $\quad[10,01,10,05,25,09,35,05,00,00,00,00,00,00,00\}$

Bronchitis $\quad\{00,00,00,10,30,05,40,05,00,00,10,00,00,00,00\}$

AAH $\{00,00,30,10,10,05,00,05,00,00,00,40,00,00,00\}$

Adeno $\{10,05,10,05,10,05,00,05,00,00,00,00,50,00,00\}$

Large cell $\{10,05,20,05,05,00,00,00,00,00,00,00,00,55,00\}$

Small cell $\{00,00,10,00,10,05,05,00,00,00,00,00,00,00,70\}$ 


\section{Computed entropies (Shannon, cellular interaction, Tsallis*) \\ Colon Adenoma \\ Colon Carcinoma \\ Shannon \\ En $=-1.012$ \\ En $=-1.609$ \\ En $=-1.737$ \\ En $=-1.488$ \\ En $=-1.638$ \\ En $=-1.637$ \\ En $=-1.330$ \\ En $=-1.099$ \\ En $=-1.494$ \\ En $=-1.579$ \\ En $=-1.094$ \\ Interaction \\ En $=-1.155$ \\ En $=-2.199$ \\ En $=-2.056$ \\ En $=-2.266$ \\ En $=-2.322$ \\ En $=-\mathbf{3 . 3 0 9}$ \\ En $=-2.929$ \\ En $=-1.515$ \\ En $=-1.872$ \\ En $=-\mathbf{2 . 4 0 2}$ \\ En $=-1.593$}

Lung normal

CAM

Pneumonia

Bronchitis

AAH:

Adeno

Large cell

Small cell

Colon normal

\section{*) q = 0.2-0.8 (between inflammatory cells, and atypical cells)}

Figure 7 Computed entropies of the model. No direct association of amount of entropy and clinical behavior of the disease was obtained.

such as cells, nuclei, mitoses, vessels, etc. In other words, an analysis of the image information results in a meaning, which is a probability function of the (predefined) diagnoses and the image information. The higher the probability the more accurate is the diagnosis. The advantage of such an algorithm is the "relatively" constancy of objects (and derived information) compared to the broad variations of images belonging to the same diagnosis $[3,8]$.

We can consider that image information is an entity that is primarily separated from the set of diagnoses. This theory induces that image information can be described as a mapping of diagnoses $M(D)$ on the image pixels $\{\mathrm{p}(\mathrm{x}, \mathrm{y}, \mathrm{g})\}$ with

$\mathrm{M}(\{\mathrm{Di}\}, \mathrm{P}) \rightarrow \mathrm{p}\{\mathrm{px}(\mathrm{x}, \mathrm{y}, \mathrm{g})\}$ with $\mathrm{p}\{\mathrm{px}(\mathrm{x}, \mathrm{y}, \mathrm{g})\}=$ maximum for the (evaluated) diagnosis $\mathrm{D}$.

Using the entropy approach we create a n-dimensional space of elementary image events and analyse the distribution in the different diseases or macrostages. It would be of formal advantage, if we could define certain elementary events that are independent from the associated meaning, i.e. independent from external knowledge. In fact, this is possible by application of stochastic geometry which has been described by Stoyan et al [24].
Naturally, one could use the pixels as elementary events and associated spectral functions in order to create the set of elementary events. However, this approach would leave us again with the problem of handling broad image variance and low probability levels.

The basic elements (or image primitives) can also be calculated by introduction of a (spatial) relationship function. It is usually called neighbourhood condition, such as Voronoi's or O'Callaghan's condition [25-27]. The simplest case is a neighbourhood function $f(x, y)$ with

$\mathrm{F}(0,1)=1$ iff $\mathrm{g}(\mathrm{x}, \mathrm{y})>$ threshold, and $\mathrm{g}(\mathrm{x}+1, \mathrm{y})>$ threshold, or $g(x, y+1)>$ threshold, i.e., two pixels are neighbors iff both of them posses a gray value above a certain predefined threshold (or within a predefined bandwidth of gray values). Naturally, a negative definition can also be applied (<threshold).

This definition allows us to define a set of primitive elements, that form an object, i.e. an elementary element of image information (object, structure, texture).

The different primitive elements include

Isolated points (i.e. pixels without neighbors)

Fibers (pixels possessing a "line" of neighboring pixels, and different start and end pixel

Circles (pixels possessing a line of neighboring pixels, and identical start and end pixel 
Plateaus (a set of pixels with a number of neighboring pixels $>2$ and connected points or lines).

Any biological meaningful object can be broken down to a set of these four primitives; for example a membrane consists of a line or a circle, a nucleus of a circle and at least one plateau, a non-completely segmented nucleus of lines, points, and plateaus, etc. .

In potential clinical application, this approach has to work with approximately 800 different macrostages (lung diseases, derived from [28], and 10,000 different features (see figure 8). To discriminate between different macrostages with a significance of $\mathrm{s}>0.95$ only 55 features per macrostage out of 1,100 available features per macrostage would be necessary.

\section{Implementation}

The selection of an appropriate threshold and/or bandwidth of the gray values as well as the image size in pixels are parameters that influence directly the implementation of this algorithm. Therefore, it has been tested on automated selected areas of interest which have been determined by analysis of texture and object features, as described elsewhere. Within the selected areas of interest the chosen threshold is of only limited influence on the expression of the elementary primitives in contrast to the whole image (see figure 9). Thus, working in correctly selected areas of interest a threshold can be chosen within a broad range without falsifying the results. On the other hand, the described technique might be useful to check the correctness of the selected field of view. An approach to finally classify diseases by the described algorithm is in preparation.

\section{Discussion}

The development of reliable and practice oriented scanners which scan whole glass slides has opened a new door in diagnostic surgical pathology or tissue - based diagnosis [1,3,5,6,29-31]. The mechanical and optical problems are in so far solved as the new canner generation can be successfully implemented into the workflow of routine diagnosis [9]. The next step waits for opening new and attractive functions of these systems. These will include the mandatory replacement and improvement of classic microscope handling, the implementation of new viewing and measurement functions, as well as the search for automated diagnosis systems. These will probably start with the implementation of so-called assistants that will guide the pathologist through all the possible tools. As in all such trends, the final aim would probably be an automated diagnosis system, which the pathologist has to control, and which might at a very later stage control itself.

\section{Statistical considerations}

- Number of potential morphology associated lung diagnoses (macrostages) $\mathbf{8 0 0}$

- Number of microstages (compartments \&

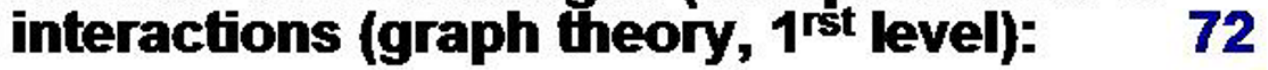
- \{(+,-)point, (+.-)fiber, (+,-)ring, (+,-)plateau\} - \{interactions (distances, neighbors)\}

- Number of features $\quad 10,000$

- Features / macrostage 1100

- Discrimination power (5\%) significance 55

\section{The algorithm possesses sufiicient power to discriminate between all known lung diseases}

Figure 8 Statistical considerations on macrostages. About 800 different lung diseases with characteristic morphology are known. Only 55 of 1,100 potential features would be needed to obtain a discrimination power $p>0.95$. 


\section{Compartments of objects}

\section{Frequency of primitives and chosen threshold Selected fields of view are less dependent F(com,thr) control function for field of view}
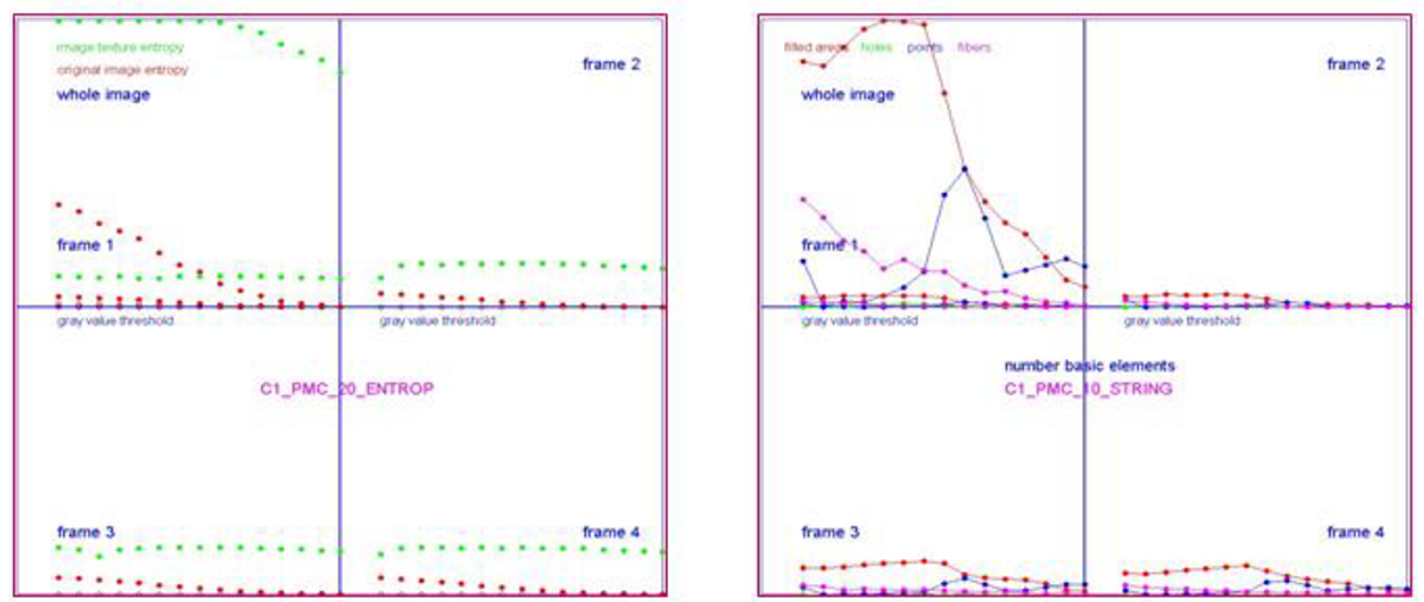

Figure 9 The generation of image primitives depends upon the chosen threshold. It remains nearly constant in correctly hosen fields of view, and can be a measure for information content in relation to the whole image.

In this article we describe only one of the possible manners to build and to implement such a system. Other algorithms have been successfully tested too [32-34]. The main idea is that we try to separate different functions that are used in the pathologist's thinking and diagnostics, and not to be confused with the contemporary application of algorithms that are in principle separated. When in the Middle Ages some genius persons tried to directly copy the flight of birds, they failed because they did not separate the upstream forces from the velocity (forward) movement. The separation of both forces induced the successful development of airplanes that have thought to be never become reality in the past.

We have shown the reader an approach that in a similar manner separates the information given in an image, and its evaluation and interpretation based upon known classification of information (diseases) by a pathologist. Having finally tested the approach, a more generalized theory of performing information into knowledge and competence in virtual microscopy is indicated.

\section{Acknowledgement}

The financial support of the Verein zur Förderung des biologisch technologischen Fortschritts in der Medizin e.V. gratefully acknowledged.
This article has been published as part of Diagnostic Pathology Volume 6 Supplement 1, 2011: Proceedings of the 10th European Congress on Telepathology and 4th International Congress on Virtual Microscopy. The full contents of the supplement are available online at

http://www.diagnosticpathology.org/supplements/6/S1.

\section{Author details}

${ }^{1}$ UICC-TPCC, Charite, Berlin, Germany. ${ }^{2}$ IAT, Heidelberg, Germany. ${ }^{3}$ BBM, Mainz, Germany. ${ }^{4}$ Institute of Pathology, University Freiburg, Freiburg, Germany.

\section{Competing interests}

The authors declare that they have no competing interests.

\section{Published: 30 March 2011}

\section{References}

1. Kayser K, Molnar B, Weinstein RS: Virtual Microscopy - Fundamentals Applications - Perspectives of Electronic Tissue - based Diagnosis. VSV Interdisciplinary Medical Publishing; 2006.

2. Weinstein RS: Innovations in medical imaging and virtual microscopy. Hum Pathol 2005, 36(4):317-9.

3. Kayser $\mathrm{K}$, et al: Towards an automated virtual slide screening: theoretical considerations and practical experiences of automated tissue-based virtual diagnosis to be implemented in the Internet. Diagn Pathol 2006, 1:10.

4. Kepper $\mathrm{N}$, et al: Visualization, Analysis, and Design of COMBO-FISH Probes in the Grid-Based GLOBE 3D Genome Platform. Stud Health Technol Inform 159:171-80.

5. Marchevsky AM, et al: The use of virtual microscopy for proficiency testing in gynecologic cytopathology: a feasibility study using ScanScope. Arch Pathol Lab Med 2006, 130(3):349-55. 
6. Merk MR, Knuechel R, Perez-Bouza A: Web-based virtual microscopy at the RWTH Aachen University: Didactic concept, methods and analysis of acceptance by the students. Ann Anat

7. Schrader T, et al: The diagnostic path, a useful visualisation tool in virtual microscopy. Diagn Pathol 2006, 1:40.

8. Kayser $\mathrm{K}$, et al: Digitized pathology: theory and experiences in automated tissue-based virtual diagnosis. Rom J Morphol Embryol 2006, 47(1):21-8.

9. Lundin $M$, et al: A European network for virtual microscopy-design, implementation and evaluation of performance. Virchows Arch 2009, 454(4):421-9.

10. Bartels P, Weber J, L D: Machine learning in quantitative histopathology. Anal Quant Cytol Histol 1988, 10:299-306.

11. Bartels PH, Vooijs GP: Vooijs: Automation of primary screening for cervical cancer. Sooner or later? Acta Cytol 43(1):7-12.

12. Gabril MY, Yousef GM: Informatics for practicing anatomical pathologists: marking a new era in pathology practice. Mod Pathol 23(3):349-58.

13. Giansanti D, et al: Virtual microscopy and digital cytology: state of the art. Ann Ist Super Sanita 46(2):115-22.

14. Kayser K: Application of structural pattern recognition in histopathology, in Syntactic and structural pattern recognition, T.P. Springer: Berlin Heidelberg New York;G. Ferraté, A. Sanfeliu, H. Bunke 1988:115-135.

15. Kayser $\mathrm{K}$, et al: Application of attributed graphs in diagnostic pathology. Anal Quant Cytol Histol 1996, 18(4):286-92.

16. Kayser $\mathrm{K}$, et al: $\mathrm{Al}$ (artificial intelligence) in histopathology-from image analysis to automated diagnosis. Folia Histochem Cytobiol 2009, 47(3):355-61.

17. Prigogine I: Introduction to Thermodynamics of Irreversible Processes. New York: John Wiley \& Sons Inc; , 2nd 1961.

18. Shannon C: A mathematical theory of communication. Bell Sys Tech J 1948, 27:379-423.

19. Pincus S: Approximate entropy as a measure of system complexity. Proc Natl Acad Sci U S A 1991, 88:2297-2301.

20. Tsallis C: Entropic nonextensivity: a possible measure of complexity. Chaos, Solitons and Fractals 2002, 13:371-391.

21. Tsekouras GA, Tsallis C: Generalized entropy arising from a distribution of q indices. Phys Rev E Stat Nonlin Soft Matter Phys 71:46-144.

22. Kayser K, Kayser G, Metze K: The concept of structural entropy in tissuebased diagnosis. Anal Quant Cytol Histol 2007, 29(5):296-308.

23. Voß K: Statistische Theorie komplexer Systeme I. EIK 1960, 3:239-244.

24. Stoyan D, Kendall WS, Mecke J: Stochastic Geomatry and its Pllications. Berlin: Akademie verlag; 1987

25. O'Callaghan J: An alternative definition for neighborhood of a point. IEEE Trans Comput 1975, 24:1121-1125.

26. Voronoi G: Nouvelles applications des paramêtres continus à la théorie des formes quadratiques, dexièmes memoire: recherches sur les parallèloedres primitifs. J Reine Angew Math 1902, 134:188-287.

27. Zahn C: Graph-theoretical methods for detecting and describing graph clusters. IEEE Trans Comput 1971, C-20:68-86.

28. Kayser K: Analytical Lung Pathology. Heidelberg, new York: Springer; 1992.

29. Kayser K, Kayser G: Virtual Microscopy and Automated Diagnosis., in Virtual Microscopy and Virtual Slides in Teaching, Diagnosis and Research., R.O. Taylor \& Francis: Boca Raton;J. Gu 2005.

30. Kumar RK, et al: Virtual microscopy for learning and assessment in pathology. J Pathol 2004, 204(5):613-8.

31. Yang $L$, et al: Virtual microscopy and grid-enabled decision support for large-scale analysis of imaged pathology specimens. IEEE Trans Inf Technol Biomed 2009, 13(4):636-44.

32. Apfeldorfer $\mathrm{C}$, et al: Object orientated automated image analysis: quantitative and qualitative estimation of inflammation in mouse lung Diagnostic Pathology 2008, 3(Suppl 1):S16.

33. Oger $M$, et al: Automated region of interest retrieval and classification using spectral analysis. Diagnostic Pathology 2008, 3(Suppl 1):S17.

34. Gilbertson J, Yagi Y: Histology, imaging and new diagnostic work-flows in pathology. Diagnostic Pathology 2008, 3(Suppl 1):S14.

doi:10.1186/1746-1596-6-S1-S9

Cite this article as: Kayser et al:: How to measure diagnosis-associated information in virtual slides. Diagnostic Pathology 2011 6(Suppl 1):S9.

\section{Submit your next manuscript to BioMed Central and take full advantage of:}

- Convenient online submission

- Thorough peer review

- No space constraints or color figure charges

- Immediate publication on acceptance

- Inclusion in PubMed, CAS, Scopus and Google Scholar

- Research which is freely available for redistribution

Submit your manuscript at www.biomedcentral.com/submit
Biomed Central 